\title{
Promoting Psychological Well-Being Through an Evidence-Based Mindfulness Training Program
}

\author{
Yi-Yuan Tang ${ }^{1 *}$, Rongxiang Tang ${ }^{2}$ and James J. Gross ${ }^{3}$ \\ ${ }^{1}$ Department of Psychological Sciences, Texas Tech University, Lubbock, TX, United States, ${ }^{2}$ Department of Psychological \\ and Brain Sciences, Washington University in St. Louis, St. Louis, MO, United States, ${ }^{3}$ Department of Psychology, Stanford \\ University, Stanford, CA, United States
}

Psychological well-being is a core feature of mental health, and may be defined as including hedonic (enjoyment, pleasure) and eudaimonic (meaning, fulfillment) happiness, as well as resilience (coping, emotion regulation, healthy problem solving). To promote psychological well-being, it is helpful to understand the underlying mechanisms associated with this construct and then develop targeted and effective training programs. In this perspective article, we discuss key components and potential brain-body mechanisms related to psychological well-being and propose mindfulness training as a promising way to improve it. Based on a series of randomized controlled trial (RCT) studies of one form of mindfulness training in adolescents and adults, the integrative body-mind training (IBMT), we use IBMT as an exemplar to provide research evidence of the positive effects of mindfulness training on psychological well-being. We focus on one of the mechanisms by which IBMT enhances psychological well-being -the interaction between mind (mindfulness) and body (bodifulness) - which involves both the central

OPEN ACCESS

Edited by:

Feng Kong,

Shaanxi Normal University, China

Reviewed by: Carmen Morawetz, Medical University of Vienna, Austria Chao Liu, Beijing Normal University, China

${ }^{*}$ Correspondence: Yi-Yuan Tang yiyuan.tang@ttu.edu

Received: 03 April 2019 Accepted: 26 June 2019 Published: 10 July 2019

Citation:

Tang $Y$-Y, Tang R and Gross JJ (2019) Promoting Psychological Well-Being Through an

Evidence-Based Mindfulness Training Program.

Front. Hum. Neurosci. 13:237. doi: 10.3389/fnhum.2019.00237 nervous system (CNS) and the autonomic nervous system (ANS). We also highlight the role of brain self-control networks, including the anterior cingulate cortex/prefrontal cortex (ACC/PFC), in improving psychological well-being. We suggest that mindfulness training may be a promising program that promotes the synergistic engagement of mind and body to achieve the goals of enhancing psychological well-being.

Keywords: emotions, psychological well-being, IBMT, mindfulness, bodifulness, quality of life, anterior cingulate cortex, striatum

\section{PSYCHOLOGICAL WELL-BEING AND HEALTH}

The importance of mental health has been increasingly emphasized in recent decades as public awareness and understanding grow. Mental health is now understood to involve both the absence of mental illness and the presence of psychological well-being. Psychological well-being is a complex construct that concerns optimal psychological functioning and experience. It may be defined as including hedonic (enjoyment, pleasure) and eudaimonic (meaning, fulfilment) happiness as well as resilience [coping, emotion regulation, healthy problem solving; Gross and Munoz, 1995; Ryff, 1995; Ryan and Deci, 2001; Community Translational Science Team (CTST), 2016; NIH Report, 2018]. Elements of psychological well-being include a sense of balance in emotion, thoughts, social relationships, and pursuits [Brown and Ryan, 2003; Community Translational Science Team (CTST), 2016; Feller et al., 2018; NIH Report, 2018], which necessitates active engagement of self-control processes such as emotion regulation. Emotion regulation is defined 
as the processes by which we influence which emotions we have, when we have them, and how we experience and express them (Gross, 1998).

Accumulating evidence has supported a causal relationship between greater psychological well-being and better overall health and improved disease-specific outcomes (Ong, 2010; Diener and Chan, 2011; DeSteno et al., 2013; Kok et al., 2013; Cohen et al., 2016). For example, changing states of well-being by increasing positive emotion and decreasing negative emotion result in salutary physiological/biological changes (e.g., inflammation, immune functioning), and contributes to diverse positive health outcomes (e.g., cardiovascular health; Kiecolt-Glaser et al., 2002; Howell et al., 2007; Diener and Chan, 2011; Feller et al., 2018).

\section{MINDFULNESS AND PSYCHOLOGICAL WELL-BEING}

Several systematic reviews and meta-analyses have suggested that psychological well-being can be enhanced by interventions such as mindfulness training (Brown and Ryan, 2003; Hutcherson et al., 2008; Weinstein et al., 2009; Hofmann et al., 2011; Goyal et al., 2014; Kong et al., 2016; Garland et al., 2017; McConville et al., 2017; Feller et al., 2018). One particular focus has been Integrative body-mind training (IBMT), which shares key components with other forms of mindfulness training, such as a systematic training of attention and self-control with an attitude of acceptance and openness to present experiences (Tang et al., 2007, 2015; Tang, 2017).

In a series of randomized controlled trials (RCTs), IBMT has shown multiple positive effects on psychological well-being and health, including increased self-control and positive emotions, and decreased negative emotions and stress hormones (Tang et al., 2007, 2015; Ding et al., 2014). In one RCT, young adults were assigned randomly to an IBMT or a relaxation training (RT) group for five sessions of brief training $(20 \mathrm{~min}$ per session). Compared to those in RT, IBMT participants showed greater improvement (from baseline to post-training) of performance in executive control (an index of self-control). IBMT participants also had lower levels of negative affect and higher levels of positive affect (Tang et al., 2007; Ding et al., 2014). In addition, IBMT participants also showed decreased stress hormone cortisol and increased immune reactivity (secretory Immunoglobulin A; Tang et al., 2007). Longer training (e.g., 20 sessions) in IBMT reduced basal stress level of cortisol and increased basal immune function, suggesting better health outcomes (Tang, 2017). To test the generalizability of these IBMT findings, we used the same RCT design in older adults and adolescents and detected similar effects on psychological well-being and health (Tang, 2009, 2017; Tang et al., 2014). In particular, following long-term IBMT practice, older adults showed significantly higher ratings in overall score of quality of life, including physical, psychological, independence and social relationships using WHOQOL-100 (Tang, 2017). Taken together, findings suggest IBMT has positive effects on psychological well-being and health.
Despite these promising findings (Chambers et al., 2009; Hölzel et al., 2011b), neurobiological studies of mindfulness training directly relating behavioral changes to brain functional activity changes remain sparse (Tang et al., 2015; Fox et al., 2016). With regard to psychological well-being, functional neuroimaging studies have yet to demonstrate a straightforward relationship between brain and behavioral improvement following mindfulness training. Nonetheless, there has been some indirect evidence showing that mindfulness capacity, a trait that is often increased after mindfulness training, can modulate neural responses to emotion-related stimuli and influence affective processing (Frewen et al., 2010; Brown et al., 2013). While correlating behavioral outcomes and alterations in brain activity and functional connectivity is somewhat challenging, given the modest sample size of typical neuroimaging studies of mindfulness training, some encouraging findings have illustrated that brain structural changes are related to improved behavioral outcomes. For instance, our RCT study on IBMT has demonstrated that improvements in white matter connectivity in anterior cingulate cortex (ACC) and PCC are correlated with enhanced positive emotion, suggesting a putative neural mechanism that underlies improvement in psychological well-being (Tang et al., 2012). Likewise, one study showed that compared to waitlist control, 2 months of mindfulness-based stress reduction induced gray matter reduction in the amygdala, which was correlated with decreased stress, suggesting an improvement in psychological well-being (Hölzel et al., 2011a; Davidson and McEwen, 2012). Together, these findings provide some evidence that mindfulness training may enhance psychological well-being through influencing brain structural plasticity. However, future investigation should focus on establishing a direct relationship between brain functional changes and behavioral improvement following mindfulness training in RCT design with a large sample size.

\section{HOW INTEGRATIVE BODY-MIND TRAINING (IBMT) WORKS}

To design effective interventions for promoting psychological well-being, we need to understand how mindfulness training such as IBMT works. Our previous work has shown increases in functional and structural plasticity of self-control networks-ACC/prefrontal cortex (PFC) and striatum support IBMT effects (Tang et al., 2009, 2010, 2012, 2015; Tang, 2017). For instance, in an RCT study with 40 college students randomly assigned to either an IBMT or RT group (20/group), the IBMT group (not RT group) had a significant cerebral blood flow increase in the (ACC; BA25, BA32), adjacent medial PFC (mPFC; BA10) and insula after a five-session training. The group $\times$ session interaction was significant for BA25 and BA10, respectively (Tang, 2017). Another RCT (IBMT vs. RT) with a large sample size also showed the stronger subgenual and adjacent ventral ACC activity and striatum activity following a five-session training in IBMT (Tang et al., 2009). These findings suggest that brief IBMT changes brain activity and functional connectivity in the ACC (Tang, 2017). 
Does longer IBMT practice induce brain structural plasticity? To test this hypothesis, we randomly assigned 45 college students into IBMT or RT groups (23:22) and delivered a 20-session training within a 4 -week period $(30$ min per session, $\sim 10 \mathrm{~h}$ in total). Using diffusion tensor imaging (DTI), we found significant increases in fractional anisotropy (an index of the white matter integrity and efficiency) in the ventral and dorsal corona radiata, an important white matter tract connecting the ACC to other structures (Tang et al., 2010). These changes were found in a band of white matter tracts connecting the ACC to striatal and cortical areas (Tang et al., 2010, 2012, 2015). To examine the time-course of white matter plasticity following IBMT, in another RCT, IBMT or RT groups received 10-20 sessions of training within 2-4 weeks. After 2-weeks of IBMT, the structural changes were mainly in axial diffusivity (an index of axonal density), while after 4-weeks both axial diffusivity and radial diffusivity (related to myelination) were improved (Tang et al., 2012; Tang, 2017). These findings indicated that IBMT induced functional and structural plasticity of self-control networks and fit well with meta-analyses of mindfulness effects on functional and structural changes (Hölzel et al., 2011b; Tang et al., 2015).

\section{THE ROLE OF BODY AND MIND}

In IBMT practice, cooperation between body and mind is emphasized in facilitating and achieving a meditation state. We thus hypothesized that one key mechanism of IBMT involves the interaction of central nervous system (CNS, brain) and autonomic nervous system (ANS, body; Tang et al., 2007, 2009, 2015). To test the hypothesis that body (physiology) and mind (brain) interaction and balance are crucial to the observed effects of IBMT, in one RCT, one group of college students was randomly assigned to experimental (IBMT) and control (RT) conditions, and received brain imaging (cerebral blood flow) and physiological measures, whereas another group of college students was randomized into IBMT and RT conditions, but underwent EEG with physiological measures. To monitor ANS activity at rest before, during, and after five 30-min sessions of IBMT or RT, in both conditions, the physiological measures included respiratory rate and amplitude, heart rate, and skin conductance response (SCR; Tang et al., 2009).

During and after the 5-session training, the IBMT group showed lower chest respiratory rate, heart rate, and SCR, but greater belly respiratory amplitude than the RT control (Tang et al., 2009), suggesting greater parasympathetic regulation of ANS. High-frequency heart rate variability (HF-HRV) is related to parasympathetic activity of the ANS and ventral ACC activation often correlates with HF-HRV, suggesting ACC regulation of parasympathetic autonomic activity (Kubota et al., 2001; Matthews et al., 2004). Compared to the same amount of RT, we also detected increased HF-HRV and frontal midline ACC theta power, suggesting greater involvement of the ANS (especially parasympathetic activity) during and after IBMT. Brain imaging showed stronger subgenual and vACC activity following IBMT and frontal midline ACC theta also correlated with HF-HRV, suggesting control by the ACC over
Improving Psychological Well-being Through Mindfulness Training (IBMT)

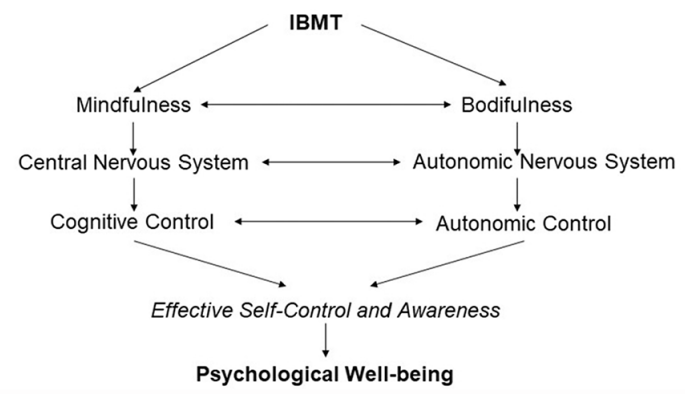

FIGURE 1 | Integrative body-mind training (IBMT) and psychological well-being.

parasympathetic activity. These findings indicate that after brief training, the IBMT shows improved ANS regulation through the self-control system such as ACC compared to the RT. This enhancement probably reflects training in the coordination and balance of body and mind by IBMT.

As shown in Figure 1, IBMT improves psychological well-being through mindfulness and bodifulness that mainly strengthen self-control ability and related CNS (i.e., ACC/mPFC and striatum) and ANS systems (i.e., parasympathetic activity; Tang et al., 2009, 2010, 2012, 2015; Kong et al., 2015). In the mindfulness field, mind or thought control is often emphasized, but the role of the body is often ignored (Kerr et al., 2013; Tang, 2017). Bodifulness refers to the gentle adjustment and exercise of body posture with a full awareness, in order to achieve a presence, balance, and integration in our bodies. For instance, bodifulness mainly involves implicit processes such as visceral or interoceptive awareness regulated by ANS. Autonomic control requires less effort and is mainly supported by the ACC/mPFC and striatum (Critchley et al., 2003; Naccache et al., 2005; Jones et al., 2015; Tang et al., 2015; Tang, 2017).

In IBMT practice, mind-body coordination and interaction are emphasized and thus significantly facilitate the training outcomes (Tang et al., 2007, 2015; Tang, 2009, 2017). Full awareness and presence of the body (bodifulness) could facilitate the mindfulness process, consistent with the literature that body posture and state affect mental processes such as emotional processing, the retrieval of autobiographical memories, and cortisol concentrations (Hennig et al., 2000; Dijkstra et al., 2007; Niedenthal, 2007; Huang et al., 2011). In early stage, mindfulness requires conscious cognitive control with effort and is supported by the dorsal lateral PFC and parietal cortex but over time, it may well involve less effort when the practice becomes more skillful, which is supported by the ACC and striatum (Tang, 2017). Cognitive control (termed as doing state) and autonomic control (termed as being state) are both key components of self-control supported by the practice and interaction of mindfulness and bodifulness, which may drive behavior and habit formation effectively (Tang et al., 2015; Tang, 2017). Taken together, if interventions such as IBMT target increases in psychological well-being through the engagement of both body and mind, effectiveness may be enhanced. 


\section{CONCLUSIONS}

A growing literature supports the idea that there is an important relationship between psychological well-being and mental and physical health in both adolescents and adults (Ong et al., 2011; DeSteno et al., 2013). IBMT has been shown to improve psychological well-being, and appears to do so via changes in self-control that are reflected in changes in both the central (brain/mind) and the autonomic (body/physiology) nervous systems. In particular, IBMT changes the state of body and mind to lead to positive outcomes in emotion, cognition, and behavior. IBMT is a paradigmatic case of how it is possible to use an evidence-based intervention that targets brain, physiology, and behavior to achieve the goals of psychological well-being and human flourishing.

In this perspective piece, we have focused on the self-control networks supporting psychological well-being following IBMT, but it should be noted that the reward system supporting motivation and positive emotion also closely links to psychological well-being (Tang et al., 2009, 2015; Tang, 2017). Future work should explore the

\section{REFERENCES}

Brown, K. W., Goodman, R. J., and Inzlicht, M. (2013). Dispositional mindfulness and the attenuation of neural responses to emotional stimuli. Soc. Cogn. Affect. Neurosci. 8, 93-99. doi: 10.1093/scan/nss004

Brown, K. W., and Ryan, R. M. (2003). The benefits of being present: mindfulness and its role in psychological well-being. J. Pers. Soc. Psychol. 84, 822-848. doi: 10.1037/0022-3514.84.4.822

Chambers, R., Gullone, E., and Allen, N. B. (2009). Mindful emotion regulation: an integrative review. Clin. Psychol. Rev. 29, 560-572. doi: 10.1016/j.cpr.2009. 06.005

Cohen, R., Bavishi, C., and Rozanski, A. (2016). Purpose in life and its relationship to all-cause mortality and cardiovascular events: a meta-analysis. Psychosom. Med. 78, 122-133. doi: 10.1097/psy.0000000000000274

Community Translational Science Team (CTST). (2016). Policy Report: Building A Public Health Model for Promoting Emotional Well-Being. Los Angeles: University of California.

Critchley, H. D., Mathias, C. J., Josephs, O., O’Doherty, J., Zanini, S., Dewar, B. K., et al. (2003). Human cingulate cortex and autonomic control: converging neuroimaging and, clinical evidence. Brain 126, 2139-2152. doi: 10.1093/brain/awg216

Davidson, R. J., and McEwen, B. S. (2012). Social influences on neuroplasticity: stress and interventions to promote well-being. Nat. Neurosci. 15, 689-695. doi: $10.1038 / \mathrm{nn} .3093$

DeSteno, D., Gross, J. J., and Kubzansky, L. (2013). Affective science and health: the importance of emotion and emotion regulation. Health Psychol. 32, 474-486. doi: 10.1037/a0030259

Diener, E., and Chan, M. Y. (2011). Happy people live longer: subjective wellbeing contributes to health and longevity. Appl. Psychol. Health Well Being 3, 1-43. doi: 10.1111/j.1758-0854.2010.01045.x

Dijkstra, K., Kaschak, M. P., and Zwaan, R. A. (2007). Body posture facilitates retrieval of autobiographical memories. Cognition 102, 139-149. doi: 10.1016/j. cognition.2005.12.009

Ding, X., Tang, Y. Y., Tang, R., and Posner, M. I. (2014). Improving creativity performance by short-term meditation. Behav. Brain Funct. 10:9. doi: 10.1186/1744-9081-10-9

Feller, S. C., Castillo, E. G., Greenberg, J. M., Abascal, P., Van Horn, R., Wells, K. B., et al. (2018). Emotional well-being and public health: proposal for a model national initiative. Public Health Rep. 133, 136-141. doi: $10.1177 / 0033354918754540$ interaction between self-control and reward systems that improve and optimize psychological well-being and mental and physical health. Moreover, different interventions may target different brain networks, and it's important to understand how different types of training programs differentially impact different brain systems and how different people might preferentially benefit from one type of intervention vs. another (Tang et al., 2015, 2016).

\section{DATA AVAILABILITY}

No datasets were generated or analyzed for this study.

\section{AUTHOR CONTRIBUTIONS}

Y-YT, RT and JG contributed to manuscript writing.

\section{FUNDING}

This work was supported by National Institutes of Health (NIH) R61AT010138.

Fox, K. C., Dixon, M. L., Nijeboer, S., Girn, M., Floman, J. L., Lifshitz, M., et al (2016). Functional neuroanatomy of meditation: a review and meta-analysis of 78 functional neuroimaging investigations. Neurosci. Biobehav. Rev. 65, 208-228. doi: 10.1016/j.neubiorev.2016.03.021

Frewen, P. A., Dozois, D. J., Neufeld, R. W., Lane, R. D., Densmore, M., Stevens, T. K., et al. (2010). Individual differences in trait mindfulness predict dorsomedial prefrontal and amygdala response during emotional imagery: an fMRI study. Pers. Individ. Dif. 49, 479-484. doi: 10.1016/j.paid.2010.05.008

Garland, E. L., Hanley, A. W., Goldin, P. R., and Gross, J. J. (2017). Testing the mindfulness-to-meaning theory: evidence for mindful positive emotion regulation from a reanalysis of longitudinal data. PLoS One 12:e0187727. doi: 10.1371/journal.pone.0187727

Goyal, M., Singh, S., Sibinga, E. M., Gould, N. F., Rowland-Seymour, A., Sharma, R., et al. (2014). Meditation programs for psychological stress and wellbeing: a systematic review and meta-analysis. JAMA Intern. Med. 174, 357-368. doi: 10.1001/jamainternmed.2013.13018

Gross, J. J. (1998). The emerging field of emotion regulation: an integrative review. Rev. Gen. Psychol. 2, 271-299. doi: 10.1037//1089-2680.2.3.271

Gross, J. J., and Munoz, R. F. (1995). Emotion regulation and mental health. Clin. Psychol. Sci. Pract. 2, 151-164.

Hennig, J., Friebe, J., Ryl, I., Krämer, B., Böttcher, J., and Netter, P. (2000). Upright posture influences salivary cortisol. Psychoneuroendocrinology 25, 69-83. doi: 10.1016/s0306-4530(99)00037-2

Hofmann, S. G., Grossman, P., and Hinton, D. E. (2011). Loving-kindness and compassion meditation: potential for psychological interventions. Clin. Psychol. Rev. 31, 1126-1132. doi: 10.1016/j.cpr.2011.07.003

Hölzel, B. K., Carmody, J., Vangel, M., Congleton, C., Yerramsetti, S. M., Gard, T., et al. (2011a). Mindfulness practice leads to increase in regional brain grey matter density. Psychiatry Res. 191, 36-43. doi: 10.1016/j.pscychresns.2010. 08.006

Hölzel, B. K., Lazar, S. K., Gard, T., Schuman-Olivier, Z., Vago, D. R., and Ott, U. (2011b). How does mindfulness meditation work? Proposing mechanisms of action from a conceptual and neural perspective. Perspect. Psychol. Sci. 6, 537-559. doi: 10.1177/1745691611419671

Howell, R. T., Kern, M. L., and Lyubomirsky, S. (2007). Health benefits: meta-analytically determining the impact of well-being on objective health outcomes. Health Psychol. Rev. 1, 83-136. doi: 10.1080/17437190701492486

Huang, L., Galinsky, A. D., Gruenfeld, D. H., and Guillory, L. E. (2011). Powerful postures versus powerful roles: which is the proximate correlate of thought and behavior? Psychol. Sci. 22, 95-102. doi: 10.1177/0956797610391912 
Hutcherson, C. A., Seppala, E. M., and Gross, J. J. (2008). Lovingkindness meditation increases social connectedness. Emotion 8, 720-724. doi: $10.1037 / \mathrm{a} 0013237$

Jones, C. L., Minati, L., Nagai, Y., Medford, N., Harrison, N. A., Gray, M., et al. (2015). Neuroanatomical substrates for the volitional regulation of heart rate. Front. Psychol. 6:300. doi: 10.3389/fpsyg.2015.00300

Kerr, C. E., Sacchet, M. D., Lazar, S. W., Moore, C. I., and Jones, S. R. (2013). Mindfulness starts with the body: somatosensory attention and top-down modulation of cortical $\alpha$ rhythms in mindfulness meditation. Front. Hum. Neurosci. 7:12. doi: 10.3389/fnhum.2013.00012

Kiecolt-Glaser, J. K., McGuire, L., Robles, T. F., and Glaser, R. (2002). Emotions, morbidity and mortality: new perspectives from psychoneuroimmunology. Annu. Rev. Psychol. 53, 83-107. doi: 10.1146/annurev.psych.53.100901.135217

Kok, B. E., Coffey, K. A., Cohn, M. A., Catalino, L. I., Vacharkulksemsuk, T., Algoe, S. B., et al. (2013). How positive emotions build physical health: perceived positive social connections account for the upward spiral between positive emotions and vagal tone. Psychol. Sci. 24, 1123-1132. doi: 10.1177/0956797612470827

Kong, F., Wang, X., Hu, S., and Liu, J. (2015). Neural correlates of psychological resilience and their relation to life satisfaction in a sample of healthy young adults. Neuroimage 123, 165-172. doi: 10.1016/j.neuroimage.2015. 08.020

Kong, F., Wang, X., Song, Y., and Liu, J. (2016). Brain regions involved in dispositional mindfulness during resting state and their relation with wellbeing. Soc. Neurosci. 11, 331-343. doi: 10.1080/17470919.2015.1092469

Kubota, Y., Sato, W., Toichi, M., Murai, T., Okada, T., Hayashi, A., et al. (2001). Frontal midline theta rhythm is correlated with cardiac autonomic activities during the performance of an attention demanding meditation procedure. Cogn. Brain Res. 11, 281-287. doi: 10.1016/s0926-6410(00)00086-0

Matthews, S. C., Paulus, M. P., Simmons, A. N., Nelesen, R. A., and Dimsdale, J. E. (2004). Functional subdivisions within anterior cingulate cortex and their relationship to autonomic nervous system function. Neuroimage 22, 1151-1156. doi: 10.1016/j.neuroimage.2004.03.005

McConville, J., McAleer, R., and Hahne, A. (2017). Mindfulness training for health profession students-the effect of mindfulness training on psychological well-being, learning and clinical performance of health professional students: a systematic review of randomized and non-randomized controlled trials. Explore 13, 26-45. doi: 10.1016/j.explore.2016.10.002

Naccache, L., Dehaene, S., Cohen, L., Habert, M. O., Guichart-Gomez, E., Galanaud, D., et al. (2005). Effortless control: executive attention and conscious feeling of mental effort are dissociable. Neuropsychologia 43, 1318-1328. doi: 10.1016/j.neuropsychologia.2004.11.024

Niedenthal, P. M. (2007). Embodying emotion. Science 316, 1002-1005. doi: $10.1126 /$ science. 1136930

NIH Report. (2018). Emotional Well-Being: Emerging Insights and Questions for Future Research. Bethesda, MD.
Ong, A. D. (2010). Pathways linking positive emotion and health in later life. Curr. Direct. Psychol. Sci. 19, 358-362. doi: 10.1177/0963721410388805

Ong, A. D., Mroczek, D. K., and Riffin, C. (2011). The health significance of positive emotions in adulthood and later life. Soc. Personal. Psychol. Compass 5, 538-551. doi: 10.1111/j.1751-9004.2011.00370.x

Ryan, R. M., and Deci, E. L. (2001). On happiness and human potentials: a review of research on hedonic and eudaimonic well-being. Annu. Rev. Psychol. 52, 141-166. doi: 10.1146/annurev.psych.52.1.141

Ryff, C. D. (1995). Psychological well-being in adult life. Curr. Dir. Psychol. 4, 99-104. doi: 10.1111/1467-8721.ep10772395

Tang, Y. Y. (2017). The Neuroscience of Mindfulness Meditation: How the Body and Mind Work Together to Change Our Behavior. Cham: Springer Nature.

Tang, Y. Y., Hölzel, B. K., and Posner, M. I. (2015). The neuroscience of mindfulness meditation. Nat. Rev. Neurosci. 16, 213-225. doi: 10.1038/nrn3916

Tang, Y. Y., Hölzel, B. K., and Posner, M. I. (2016). Traits and states in mindfulness meditation. Nat. Rev. Neurosci. 17:59. doi: 10.1038/nrn.2015.7

Tang, Y. Y., Lu, Q., Fan, M., Yang, Y., and Posner, M. I. (2012). Mechanisms of white matter changes induced by meditation. Proc. Natl. Acad. Sci. U S A 109, 10570-10574. doi: 10.1073/pnas.1207817109

Tang, Y. Y., Lu, Q., Geng, X., Stein, E. A., Yang, Y., and Posner, M. I. (2010). Shortterm meditation induces white matter changes in the anterior cingulate. Proc. Natl. Acad. Sci. U S A 107, 15649-15652. doi: 10.1073/pnas.1011043107

Tang, Y. Y., Ma, Y., Fan, Y., Feng, H., Wang, J., Feng, S., et al. (2009). Central and autonomic nervous system interaction is altered by short-term meditation. Proc. Natl. Acad. Sci. U S A 106, 8865-8870. doi: 10.1073/pnas.0904031106

Tang, Y. Y. (2009). Exploring the Brain, Optimizing the Life. Beijing: Science Press.

Tang, Y. Y., Ma, Y., Wang, J., Fan, Y., Feng, S., Lu, Q., et al. (2007). Short-term meditation training improves attention and self-regulation. Proc. Natl. Acad. Sci. U S A 104, 17152-17156. doi: 10.1073/pnas.0707678104

Tang, Y. Y., Tang, R., Jiang, C., and Posner, M. I. (2014). Short-term meditation intervention improves self-regulation and academic performance. J. Child Adolesc. Behav. 2:154. doi: 10.4172/2375-4494.1000154

Weinstein, N., Brown, K. W., and Ryan, R. M. (2009). A multi-method examination of the effects of mindfulness on stress attribution, coping and emotional well-being. J. Res. Pers. 43, 374-385. doi: 10.1016/j.jpp.2008.12.008

Conflict of Interest Statement: The authors declare that the research was conducted in the absence of any commercial or financial relationships that could be construed as a potential conflict of interest.

Copyright $\odot 2019$ Tang, Tang and Gross. This is an open-access article distributed under the terms of the Creative Commons Attribution License (CC BY). The use, distribution or reproduction in other forums is permitted, provided the original author(s) and the copyright owner(s) are credited and that the original publication in this journal is cited, in accordance with accepted academic practice. No use, distribution or reproduction is permitted which does not comply with these terms. 